\title{
Inhalation Health Risk Assessment for the Human Tracheobronchial Tree under PM Exposure in a Bus Stop Scene
}

\author{
Xiaoyu Xu ${ }^{1,2,3}$, Yidan Shang ${ }^{2}$, Lin Tian ${ }^{2 *}$, Wenguo Weng ${ }^{1}$, Jiyuan $\mathrm{Tu}^{2,3,4^{*}}$ \\ ${ }^{1}$ Institute of Public Safety Research, Department of Engineering Physics, Tsinghua University, Beijing 100084, China \\ ${ }^{2}$ School of Engineering - Mechanical and Automotive, RMIT University, Bundoora, VIC 3082, Australia \\ ${ }^{3}$ School of Mechanical and Manufacturing Engineering, University of New South Wales, Sydney, NSW 2052, Australia \\ ${ }^{4}$ Key Laboratory of Ministry of Education for Advanced Reactor Engineering and Safety, Institute of Nuclear and New \\ Energy Technology, Tsinghua University, Beijing 100084, China
}

\begin{abstract}
Inhalation exposure to airborne particulate matter (PM) can induce respiratory/cardiovascular disease and lung cancer in humans. Determining the specific particle deposition distribution in the human tracheobronchial tree is crucial to evaluating the health risk. Thus, an integrated human nasal-oral-tracheobronchial airway model was employed to study the particle deposition, and empirical equations for calculating the lung lobe risk contribution fractions were developed. The risk contribution of each lobe to non-carcinogenesis and carcinogenesis was predicted using prior experimental data collected at a bus stop. The regional inhalation health risk was analyzed by evaluating the hazard quotient (HQ) and excess lifetime cancer risk (ELCR) of selected non-carcinogenic and carcinogenic elements (viz., $\mathrm{Cr}, \mathrm{Mn}$, and Ni). Fine particles $(10 \mathrm{~nm}-1 \mu \mathrm{m})$ contributed the highest risk fractions for the lung lobes, inducing higher potential health consequences in the lungs than coarser particles. Cr posed carcinogenic lung risks to people who commuted by public transport, with the ELCR to every lobe exceeding the recommended limit. The non-carcinogenic and carcinogenic risks were 1.5 times greater for the right lung than for the left lung. Of the lung lobes, the RLL incurred the highest risk, followed by the LLL, RUL, LUL, and RML. Inhalation exposure to $\mathrm{Cr}$ posed a much higher risk to the lungs than exposure to Ni and Mn. However, compared to the other two elements, Mn potentially induced a higher chance of developing upper respiratory disease.
\end{abstract}

Keywords: Tracheobronchial airway; Particulate matter; Chemical composition; Inhalation assessment; Lung lobe deposition.

\section{INTRODUCTION}

Inhalation exposure to airborne particulate matter (PM) has been recognized as a major trigger of the increased morbidities and mortalities in acute and/or chronic health conditions (e.g., respiratory/cardiovascular diseases and lung cancer; Baxter et al., 2010; Donaldson et al., 2001; Turner et al., 2011). Particulate matter, including nanoparticles and microparticles, is highly respirable into human respiratory system. Particle deposition in human respiratory airways could induce health risk to human. Local accumulations of particles in the bronchial airway bifurcations, which have been shown to be apparent sites of neoplastic lesions, might play a key role in triggering lung cancer (Balásházy

\footnotetext{
* Corresponding authors.

Tel.: +61 399256093

E-mail address: lin.tian@rmit.edu.au (L. Tian); jiyuan.tu@rmit.edu.au (J. Tu)
}

et al., 2003). Fine particles are more likely to pass through nasal and/or oral barriers and penetrate to lower airways or even into the lungs ( $\mathrm{Xu}$ et al., 2018). It is of great significance to study the detailed particle deposition distribution in human tracheobronchial airways and evaluate the potential inhalation health risk in the lungs.

Over the past decade, computational fluid dynamics (CFD) simulations have been carried out to better understand the particle deposition distribution in human tracheobronchial airways. This saved significant time and cost compared to in vitro experiments (Lizal et al., 2015; Tian and Ahmadi, 2016; Deng et al., 2018). A 16-generation coplanar symmetric tracheobronchial airway, based on the Weibel model (Weibel, 1963), was employed for predicting nanoparticle deposition (Zhang et al., 2008). The study illustrated that the geometric effects, including daughterbranch rotation, was minor for nanoparticles with $d_{p}>10 \mathrm{~nm}$. In another study, micro- and nanoparticle transportation and deposition in an asymmetric multi-level human lung bifurcation model were applied, which suggested that the branching angles of the airway bifurcation model were 
important parameters for particle deposition (Tian and Ahmadi, 2012). With the development of CT scanning technology, a realistic human upper lung airway was reconstructed from CT scanned images (Inthavong et al., 2010) and local "hot-spots" where particle deposition was concentrated were identified. The study suggested that age could have a significant influence on particle deposition in TB regions. For adults, particles were more likely to deposit in the lower airways (G9-G12) than in the upper (G3-G6), as gravitational sedimentation was the dominant deposition mechanism (Deng et al., 2018). Most of these airway models only contained trachea and the upper bronchial tree airways. However, Kim et al. (2018) suggested that upper airways affected the flow and ventilation distribution in the distal airways. It was essential to utilize a complete respiratory airway to better understand the particle deposition distribution in lung bifurcations.

The chemical composition and metal speciation in particulate matter were suggested to play a crucial role in assessing human health risks of PM exposure (Bell et al., 2007; Sah et al., 2017). Toxic metals combined with particulate matter may enter the body through oral and nasal inhalation, ingestion and skin contact (Kong et al., 2011). Franklin et al. (2008) illustrated that the chemical species, such as aluminum (Al), arsenic (As), silicon ( $\mathrm{Si}$ ), nickel (Ni) and sulfate could aggravate the association between $\mathrm{PM}_{2.5}$ exposure and mortality, and the PM mass alone was not an adequate metric for assessing the health impact. Many other metals, such as cadmium $(\mathrm{Cd})$, chromium $(\mathrm{Cr})$, lead $(\mathrm{Pb})$, manganese $(\mathrm{Mn})$, copper $(\mathrm{Cu})$, vanadium $(\mathrm{V})$ and zinc $(\mathrm{Zn})$ were also considered to be commonly present toxicants in particulate matter (Loxham et al., 2013; Zhai et al., 2014). Although work has been done on evaluating the PM inhalation exposure in various contexts, such as on the roof of a mining and metallurgical city (Zheng et al., 2018), on the road (Ferreira-Baptista and De Miguel, 2005), in an informal electronic-waste recycling site (Zheng et al., 2016) and on the stairways (Zheng et al., 2010), most of them were limited on evaluating the health risks of the total respiratory system. Non-cancer and cancer inhalation health risks in the whole respiratory tract were analyzed in roadway, light rail and subway transit routes, which suggested the best option for commuters was to use above-ground light-rail transportation (Lovett et al., 2018). The deposition distributions of particles and selected non-carcinogenic and carcinogenic elements in the human respiratory system were analyzed by employing the existing respiratory deposition models at a bus stop, alongside a highway, and at an industrial site in Singapore (You et al., 2017), and the hazard quotient (HQ) and excess lifetime cancer risk (ELCR) for the elements were used to evaluate the total respiratory system inhalation health risk, following the United States Environmental Protection Agency (EPA) guidance.

Few studies were conducted to investigate the detailed respiratory regional risks by considering particle deposition distributions. Nasal cavity and olfactory dosages in a wirecut electrical discharge machine shop (Tian et al., 2017) and the dosages in a realistic firefighter nasal cavity, middle turbinate, middle meatus, onto the face and penetrated into the lower airways in a composite deck fire (Xu et al., 2018) were estimated by combining experimental and numerical studies. Lung health risks were analyzed by evaluating the doses of deposited particles in the lung from six sources (Vu et al. 2016). Traffic emissions were reported as the main source of particle number deposition in both the regional and total lung, while secondary aerosols were the main source of surface area and volume deposition in the lung. Although human health risk assessment was a complicated process due to the lack of accurate knowledge, changing circumstances, and inter-personal variations, it was a beneficial tool to evaluate the likelihood of adverse health effects (carcinogenic and non-carcinogenic) (Kong et al., 2011). According to the U.S. EPA (2009), 100\% of the dosimetry in the respiratory tract was available to enter the systemic circulation (remote toxicity), or to interact locally at the respiratory tract (local toxicity). EPA suggested that Inhalation Unit Risk (IUR) and Reference Concentration (RfC) for an inhaled chemical contaminant should be employed in inhalation health risk assessment. The estimated target hazard quotient and the excess lifetime cancer risk were based on the total respiratory and extra-respiratory dosimetry. The targeted regional risk assessment, such as lung cancer, should be further investigated.

In this study, nano- and microparticle deposition in human tracheobronchial regions were simulated in an integral human nasal-oral-tracheobronchial airway model. The model was reconstructed from CT scanned images, accounting for facial features and the external environment. Non-carcinogenic and carcinogenic elements $(\mathrm{Cr}, \mathrm{Mn}, \mathrm{Ni})$ were considered in the study. A method for regional (lung lobes) health risks assessment, by evaluating the hazard quotient (HQ) and the excess lifetime cancer risk (ELCR) of the selected elements, was proposed. The particle size distribution and chemical composition concentrations were obtained from the experimental data of a bus stop (You et al., 2017). The equations of risk contribution fraction in each lobe were developed. The contribution of each lobe to non-carcinogenesis and carcinogenesis risks were discussed under the bus stop scenario.

\section{METHODS}

\section{Nasal-Oral-Tracheobronchial Airway Model}

A realistic integrated anatomical model of the human respiratory tract, containing major tracheobronchial tree and facial features, was reconstructed from CT images of a 43-year-old healthy male via segmentation technique. The realistic human face was exposed to the external surroundings with varied chemical compositions of particulate matter. Nasal cavity, oral cavity, pharynx, larynx, trachea, and bronchial tree were all included in the model (Fig. 1(a)). The tracheobronchial tree (TB) is anatomically divided into 5 lobes (Schünke et al., 2006), including 5 lobar bronchus and 20 segmental bronchi according to the anatomy (Fig. 1(b)). Apical (I), posterior (II) and anterior (III) segmental bronchi belonged to the right upper lobe (RUL); lateral (IV) and medial (V) segmental bronchi were part of the right middle lobe (RML); superior (VI), medial basal(VII), 
(a)

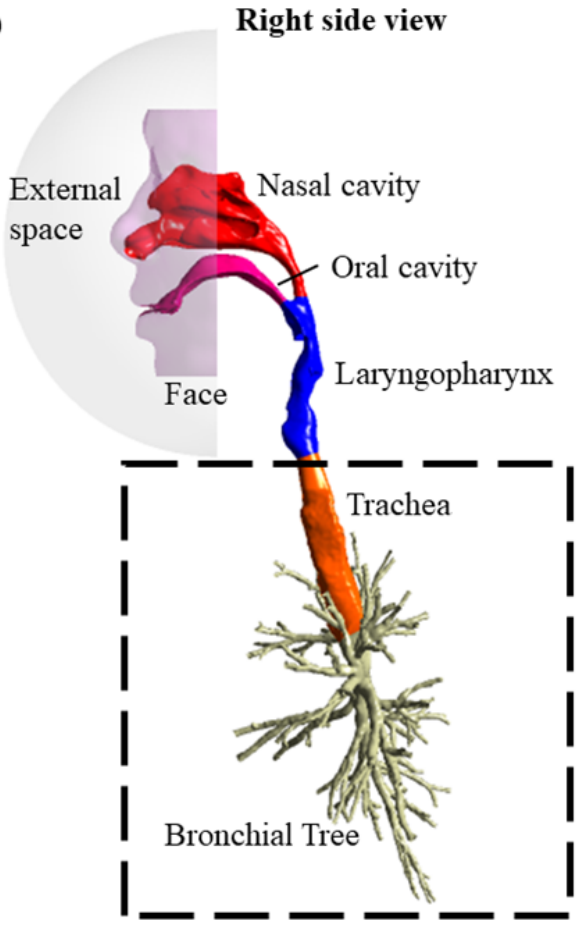

(b)

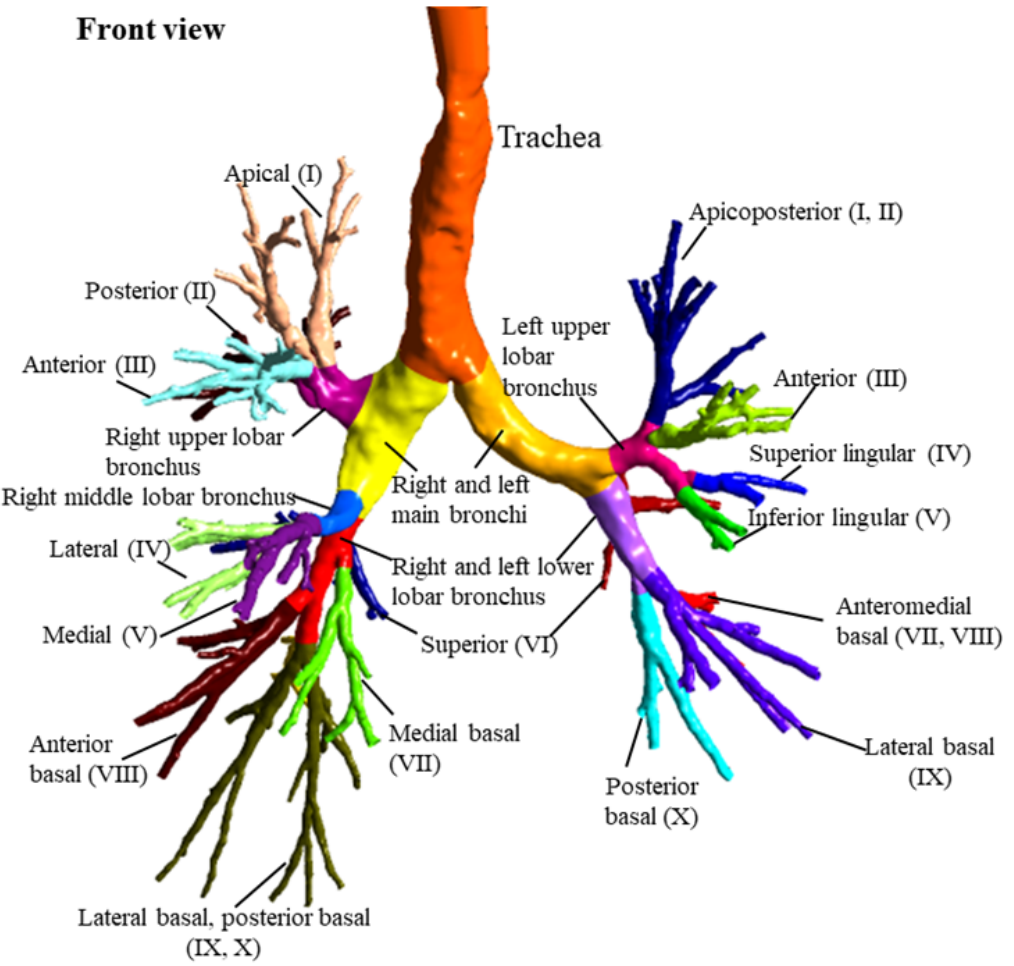

Fig. 1. Schematic of the human airway geometric model: (a) anatomical realistic human nasal-oral-tracheobronchial airway model reconstruction (CT scans); (b) detailed division of tracheobronchial airway model.

anterior basal (VIII), lateral basal (IX) and posterior basal (X) segments were classified as the right lower lobe (RLL). The left upper lobe (LUL) included apicoposterior (I, II), anterior (III), superior lingular (IV) and inferior lingular (V) segments; the left lower lobe (LLL) consisted of superior (VI), medial basal (VII), anterior basal (VIII), lateral basal (IX) and posterior basal (X) segmental bronchi. In the current study, particles were uniformly released on a hemisphere (of radius $3 \mathrm{~cm}$ ) from the center of the nose and oral and traveled from the external space, via nostrils and mouth cavity to the end of the segmental bronchi.

ICEM CFD (v18.0; ANSYS) was used to generate the computational mesh (Fig. 2). Polyhedron meshing scheme was employed and 5 prism layers were applied to the bounding respiratory walls to resolve the viscous sub-layer. The resulting mesh consisted of 2,872,136 cells and 13,089,220 faces. Mesh independency was tested and achieved. The first grid point away from the wall surface was given in wall units of $y^{+} \leq 1.0$. A workstation with 64 GB RAM, 4 GB memory of graphics card and 20-core processor was used for the simulations.

\section{Fluid Flow Simulation and Particle Tracking}

The current study employed a steady inhalation model, assuming that the deposition mainly occurred during the inspiration process (Se et al., 2010). The breathing pattern was shown to affect deposition for micron range of particles between 1 and $5 \mu \mathrm{m}$; however, the effect of nanoparticle deposition was not fully understood (Häußermann et al., 2002). A moderate steady breathing of $18 \mathrm{~L} \mathrm{~min}^{-1}$ through both nasal and oral was considered in this study. At this flow rate in the respiratory tract, airflow was laminar and transition state. Transitional Shear Stress Transport (SSTtransitional) model was employed. It was suitable to model the internal respiratory flow, which gave a good prediction of turbulence kinetic energy and predict mean velocity distributions accurately for laminar-transitional-turbulent flow (Zhang and Kleinstreuer, 2011; Inthavong et al., 2012). The SST-transitional model was not repeated here for brevity (Menter et al., 2006).

The airflow was modeled using ANSYS Fluent v18.0. Second-order upwind schemes and SIMPLE method were used to improve the computational accuracy and handle the pressure-velocity coupling. The pressure of the external surrounding was set as the atmospheric pressure. A pressure difference was enforced between the external environment and end of the segmental bronchus to initiate the inhalation. The flow rate weighting in each of the five lobes was determined by the corresponding lobar volume fractions (Horsfield et al., 1971): 20\% for RUL, 10\% for RML, 25\% for RLL, $20 \%$ for LUL, and 25\% for LLL.

Lagrangian particle tracking was employed for particle trajectories. Neglecting collision between particles and assuming the particles to be spherical, the particle equation is:

$$
\frac{d u_{p}}{d t}=\frac{1}{C_{c}} F_{D}+\frac{g\left(\rho_{p}-\rho_{g}\right)}{\rho_{p}}+F_{s}+F_{B}
$$

where $u_{p}, \rho_{p}, t$ and $\rho_{g}$ are the particle velocity, particle density, time and the air density. $g$ is the gravitational 

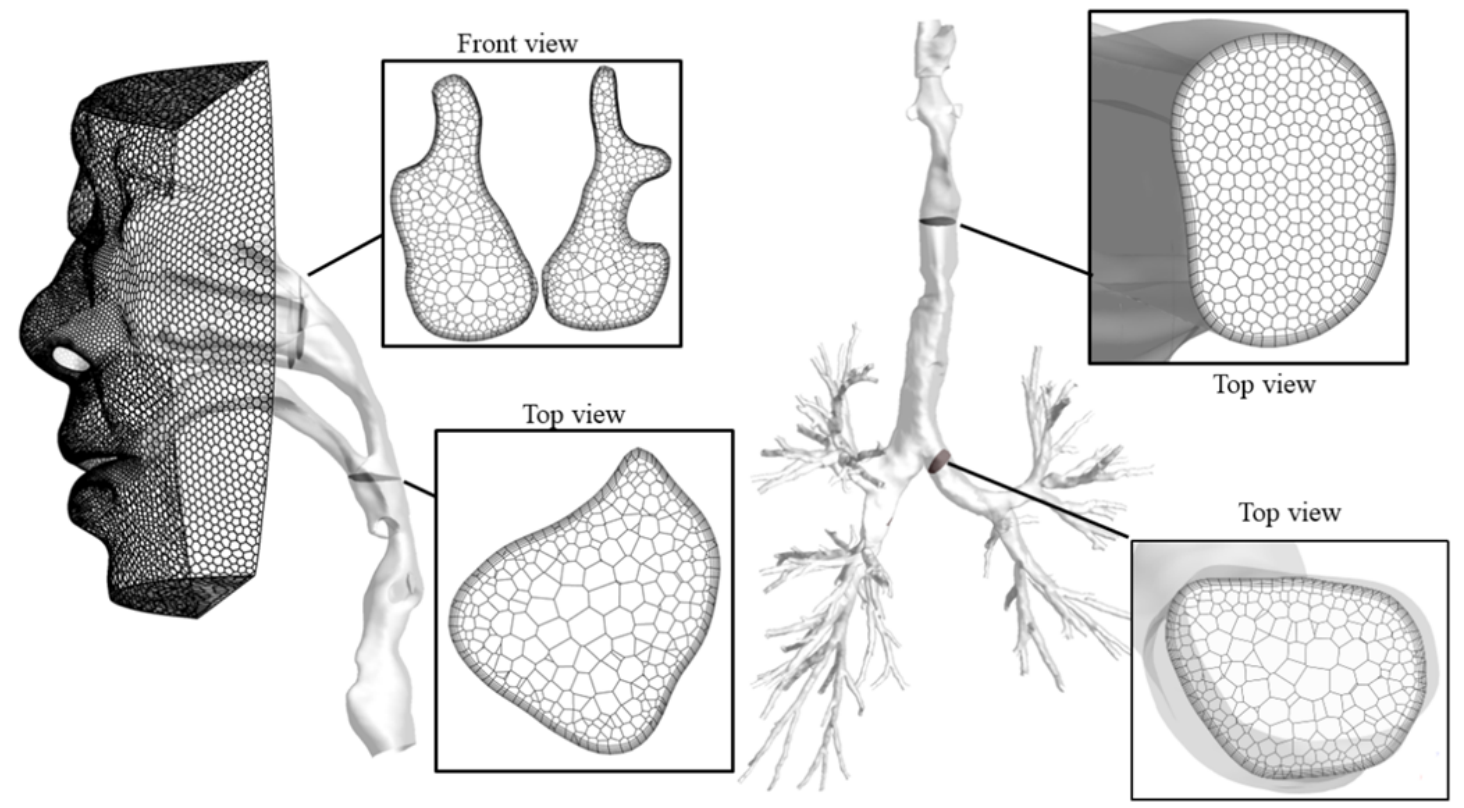

Fig. 2. Computational mesh with polyhedron and prism layers meshing schemes.

constant. $F_{D}$ is the drag given as:

$$
F_{D}=\frac{18 \mu_{g}\left(u_{g}-u_{p}\right)}{\rho_{p} d_{p}^{2}}
$$

where $d_{p}$ is the particle diameter and $\mu_{g}$ is the molecular viscosity of the air. $C_{c}$ is the Cunningham correction, given as:

$$
C_{c}=1+\frac{2 \lambda}{d_{p}}\left(1.257+0.4 e^{\left(-1.1 d_{p} / 2 \lambda\right)}\right)
$$

$\lambda$ is the molecular mean free path of air. $F_{S}$ in Eq. (1) is the Saffman lift force and $F_{B}$ is the Brownian diffusion force with the amplitudes of $\zeta \sqrt{\pi S_{0} / \Delta t}$. $\zeta$ is zero-mean, unitvariance independent Gaussian random numbers, $\Delta t$ is the time step for particle integration and $S_{0}$ is a spectral intensity function:

$$
S_{0}=\frac{216 v_{g} k_{B} T}{\pi^{2} \rho_{g} d_{p}{ }^{5}\left(\frac{\rho_{p}}{\rho_{g}}\right)^{2} C_{c}}
$$

where $k_{B}$ is the Boltzmann constant equal to $1.38 \times 10^{-23}$ $\mathrm{J} \mathrm{K}^{-1}, v_{g}$ is the air kinematic viscosity and $T$ is the absolute temperature of the inspiratory air in the respiratory tract. For micron particles, the Cunningham correction in Eq. (1) is approaching 1 and Brownian diffusion forces can be neglected.

The simulation was carried out with ANSYS FLUENT 18.0 discrete phase model (DPM). Particle dispersion was modeled until it either escaped from the airway, or trapped onto respiratory walls when the particle was within radius distance away from the airway surface. A homogeneous dispersion of the particulate matter was assumed in the breathing zone. In the present study, particles were uniformly released in a hemispheric profile from the nasal and oral entrance. Approximately 90,000 independents, monodisperse airborne particles for 1, 1.2, 2, 5, 10, 20, 30, 50, 70, 100, $150,300,500 \mathrm{~nm}$ and 1, 2, 3, 5, 7, 10, $15 \mu \mathrm{m}$ were included in the study. In the current simulation, particle densities of 7190,7440 and $8908 \mathrm{~kg} \mathrm{~m}^{-3}$, resembling that of $\mathrm{Cr}, \mathrm{Mn}$, and $\mathrm{Ni}$, were used.

\section{Deposition and Risk Contribution Fraction}

Particle deposition in human respiratory airways can be quantified in terms of deposition fraction $\left(D F_{p}\right)$, defined as:

$D F_{p}=\frac{N_{d}}{N_{t}} \times 100 \%$

where $N_{d}$ is the number of trapped particles in a specific region, $N_{t}$ is the number of particles entering human nasal and oral. It is an important parameter characterizing the regional filtering capacity, the fate of the inhaled particles and particle penetration capacity. Deposition fraction is closely related to the transport mechanisms and for nanoparticles, size, diffusivity and airflow rate are the key parameters. For microparticles, particle size, density and airflow rate are the governing parameters affecting the transport outcome. Due to the geometric complexity of human airways, no analytical expression is available to calculate the deposition fraction. Empirical fitted equations are frequently used to develop the deposition fraction as a function of the governing parameters.

In the simulation, particles in the trachea-bronchial tree 
either deposited on the surface of the bronchus airways or escaped from the outlets of the segmental bronchus. It was assumed that all deposited particles contributed to inducing lung cancer or cancer at remote sites through systemic circulation. Risk contribution fraction ( $\mathrm{RCF}$ ) was used to evaluate the carcinogenic potential in lung and in each lobe. Empirical fitted equations could also be used to develop the risk contribution fraction as a function of the governing parameters:

$R C F=\frac{N_{d}+N_{o}}{N_{t}} \times 100 \%$

where $N_{o}$ is the number of escaped particles from the specific region segmental bronchus outlet.

Airborne contaminants contain polydisperse particles where the volume size distribution (volume of particles per unit volume) can be expressed as $V\left(d_{p}, \vec{r}\right)\left(\mu \mathrm{m}^{3} \mathrm{~cm}^{-3}\right)$. Here $\vec{r}$ is position vector and $d_{p}$ is particle diameter $(\mathrm{nm})$. Assuming the heavy metal in PM all followed the same volume size distribution, the volume size concentration can be expressed as $\mathrm{V}\left(C, d_{p}, \vec{r}\right)$, where $C\left(\mathrm{ng} \mathrm{m}^{-3}\right)$ is the average heavy metal concentration in PM. The heavy metal particle risk contribution concentration $C_{\text {contri }}\left(\mu \mathrm{g} \mathrm{m}^{-3}\right)$ can be calculated as:

$$
C_{\text {contri }}=\int_{d_{p_{1}}}^{d_{p_{2}}} \rho_{p} V\left(C, d_{p}, \vec{r}\right) \cdot(R C F) \cdot d\left(d_{p}\right)
$$

\section{Health Risk Assessment}

PM exposure at a bus stop scenario was utilized in this study (You et al., 2017). The measured experimental data was collected at a bus stop outside the Lee Kong Chian Natural History Museum of the National University of Singapore by using the aerosol spectrometer and cascade impactor. The field particle data were obtained by averaging the data from two sampling stations, which was around $1 \mathrm{~m}$ away from the curb, $8 \mathrm{~m}$ apart at the two ends (front and back) of the bus stop and $1.5 \mathrm{~m}$ in height, indicating the human breathing zone. The climate in August in Singapore was hot and humid. The surrounding temperature and the respiratory temperature were assumed to be the same, at $37^{\circ} \mathrm{C}$. Normalized particle volume size distribution at the bus stop was fitted by a lognormal equation:

$$
\begin{aligned}
V\left(d_{p}, \vec{r}\right) & =13.8 e^{\left.-\left(\left(\ln d_{p}-\ln 273.1\right) / \sqrt{2} \ln 1.34\right)^{2}\right)} \\
& +8 e^{\left.-\left(\left(\ln d_{p}-\ln 6039\right) / \sqrt{2} \ln 2.92\right)^{2}\right)}
\end{aligned}
$$

The averaged size-dependent concentrations $(C)$ of selected heavy metals in the ambient environment were shown in Table 1 (You et al., 2017). The risk assessment of inhalation exposure to the selected elements (i.e., $\mathrm{Cr}$, $\mathrm{Mn}, \mathrm{Ni}$ ) in human TB regions was performed based on the U.S. EPA guidance (2009). The regional inhalation exposure is estimated as:

$$
E C_{\text {contri }_{i}}=C_{\text {contri }} \cdot E T \cdot E F \cdot E D / A T n
$$

where $E T$ (hours day ${ }^{-1}$ ), $E F$ (days year ${ }^{-1}$ ), $E D$ (years) are the exposure time, frequency and duration, respectively. $A T n$ is the averaging time of exposure. For non-carcinogens, $A T n=E D \times 365$ days $\times 24 \mathrm{~h} \mathrm{day}^{-1}$, while for carcinogens, $A T n=70$ years $\times 365$ days $\times 24 \mathrm{~h} \mathrm{day}^{-1}$. For the bus stop scenario, ET,EF,ED are $1 / 3 \mathrm{~h} \mathrm{day}^{-1}, 300$ days year $^{-1}$, and 30 years, respectively.

The exposure risk to heavy metals in TB section through inhalation can be estimated by the hazard quotient (HQ) and excess lifetime cancer risk (ELCR) given as:

$$
\begin{aligned}
& H Q_{i}=E C_{\text {contri }_{i}} /\left(R f C_{i} \times 1000 \mu \mathrm{g} \mathrm{m}^{-3}\right) \\
& E L C R_{i}=I U R_{i} \times E C_{\text {contri }_{i}}
\end{aligned}
$$

where $H Q_{i}$ is the hazard quotient in the study region, $E L C R_{i}$ is excess lifetime carcinogenic risk in the specific region, $R f C_{i}\left(\mathrm{mg} \mathrm{m}^{-3}\right)$ is the inhalation reference concentration, $I U R_{i}\left(\left(\mu \mathrm{g} \mathrm{m}^{-3}\right)^{-1}\right)$ is the inhalation unit risk (U.S. EPA, 2018). $H Q=1$ suggests a significant noncarcinogenic health risk. $E L C R_{i}$ suggests the probability of lung cancer due to exposure to specific metal contaminants for 70 years. When $E L C R_{i}$ exceeds $1 \times 10^{-6}$, it indicated the higher chance to develop lung cancer.

Since health risk assessment concerns long-term outcome, all the risk contribution fraction (RCF) was estimated under normal human breathing of $18 \mathrm{~L} \mathrm{~min}^{-1}$ through both nasal and oral airways in current study. The risk contribution (RC) was used to indicate the chance of each lobe contributing to non-carcinogenic and carcinogenic consequences. It is given as:

$$
R C_{i}=H Q_{i} / H Q=E L C R_{i} / E L C R=C_{\text {contri }_{i}} / C
$$

\section{RESULTS AND DISCUSSION}

\section{Validation}

Particle deposition in the trachea against the inertial parameter between present study and prior experiments (Schlesinger et al., 1977; Zhou and Cheng, 2005) were given in Fig. 3. The inertial parameter $\left(\mathrm{mm}^{2} \mathrm{~g} \mathrm{~s}^{-1}\right), I$, relevant to the micron-sized particles including particle

Table 1. Size-dependent heavy metal concentration in the bus stop scenario (You et al., 2017).

\begin{tabular}{llllll}
\hline $\mathrm{C}\left(\mathrm{ng} \mathrm{m}^{-3}\right)$ & $2.5-10 \mu \mathrm{m}$ & $1.0-2.5 \mu \mathrm{m}$ & $0.5-1.0 \mu \mathrm{m}$ & $0.25-0.5 \mu \mathrm{m}$ & $0-0.25 \mu \mathrm{m}$ \\
\hline $\mathrm{Cr}$ & 3.28 & 5.37 & 9.13 & 1.35 & 31.47 \\
$\mathrm{Mn}$ & 2.71 & 0.84 & 0.87 & - & 0.74 \\
$\mathrm{Ni}$ & 9.33 & 20.35 & 25.71 & 2.43 & 12.48 \\
\hline
\end{tabular}




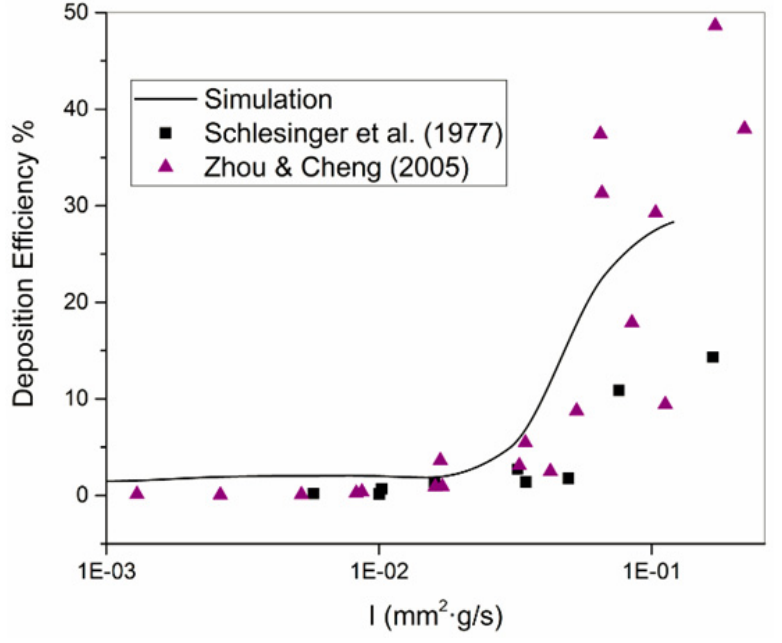

Fig. 3. Comparison of the simulated particle deposition fraction in the trachea with in vitro measurement (Schlesinger et al., 1977; Zhou and Cheng, 2005).

mass, particle diameter, and the averaged fluid momentum, is defined as:

$$
I=\rho_{p} d_{p}^{2} Q
$$

where $Q$ is the volume flow rate $\left(\mathrm{cm}^{3} \mathrm{~s}^{-1}\right)$. The deposition efficiency $\left(D E_{p}\right)$ in the trachea is given as:

$D E_{p}=\frac{N_{d}}{N_{r}} \times 100 \%$
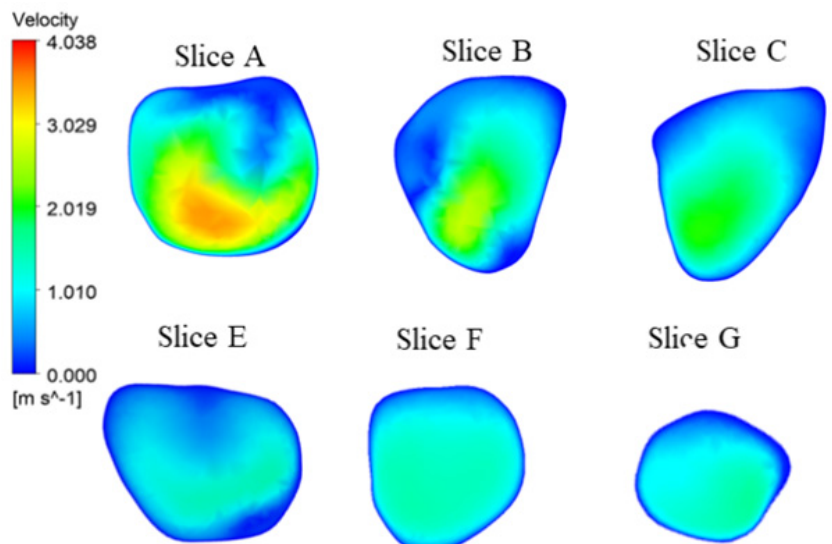

Slice F

Slice G
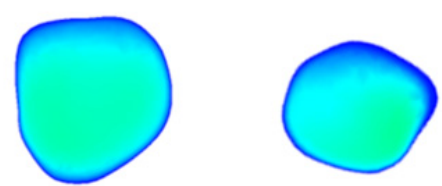

Slice I

Slice J
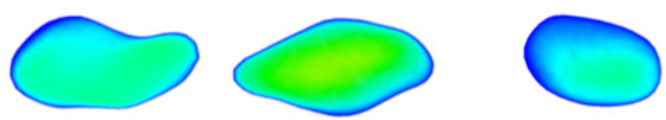

where $N_{r}$ is the number of particles entering the trachea. The simulated particle deposition efficiencies in the trachea as a function of the inertial parameter in nasal-oraltracheobronchial model agreed well in the trend with the experimental measurements. The trachea deposition efficiency stabilized around $1 \%$ and then sharply increased when the inertial parameter reaches $0.02 \mathrm{~mm}^{2} \mathrm{~g} \mathrm{~s}^{-1}$. The deposition fraction discrepancies between the simulation and experiments might be attributed to the variation in morphometry. The experimental models only contained trachea and bronchial airways, and do not include an oral or nasal cavity. It was suggested that the upper airways affected the flow and ventilation distribution in the distal airways (Kim et al., 2018). Overall our results for particle deposition in trachea were reasonable.

\section{Tracheobronchial Air Flow Patterns}

The streamlines and velocity contours at selected cross sections in the tracheobronchial airways of the nasal-oraltracheobronchial model were shown in Fig. 4. Breathing rate was $18 \mathrm{~L} \mathrm{~min}^{-1}$ through both nasal and oral openings. Slice A, B, and C were on the trachea. Slice D and J were on the right main bronchi, $\mathrm{E}$ and $\mathrm{F}$ were on the left main bronchi. Slice F, H, I, K, and L were on the left upper lobar bronchus (LULB), left lower lobar bronchus (LLLB), right upper lobar bronchus (RULB), right middle lobar bronchus (RMLB), and right lower lobar bronchus (RLLB), respectively. All cross-sections were viewed from the perspective of the main flow. The Reynolds number in Slice A was about 2000. The velocities were gradually decreasing from the inlet of the trachea to the outlets of the segmental bronchus due to partition at multiple bifurcations. Flow paths were indicated by bulk air passages and peak

Fig. 4. Airflow streamline and velocity contours at selected cross sections in tracheobronchial airways through nasal and oral breathing $\left(18 \mathrm{~L} \mathrm{~min}^{-1}\right)$. 
velocity distributions. Bulk air was largely found in the anterior rather than the posterior of the trachea. Then the velocities approached steady and uniform in the LULB, LLLB, RULB, and RMLB. In the RLLB, a velocity jet was formed due to the larger volume of air flow went through the narrowing cross-sections. Air flows changed directions in each bifurcation and velocities were found higher near the inner wall of the bifurcations than the outer sides. Peak velocities and changing of flow directions indicated that the breathing air was more likely to be impacted onto the surrounding walls. The transport of inhaled air in tracheobronchial airways provided insight into the understanding of airborne particle movement and deposition, as the air carried and transported these particles.

\section{Particle Risk Contribution Fraction Equations in Tracheobronchial Airways}

Fig. 5 presented the simulated $\mathrm{Cr}$ particle $\left(7190 \mathrm{~kg} \mathrm{~m}^{-3}\right.$, $1 \mathrm{~nm}-10 \mu \mathrm{m})$ risk contribution fraction against the particle diameter in the left lung (LL), right lung (RL), trachea and main bronchi (T\&M), LLL, LUL, RUL, RML, and RLL, respectively. It was shown from the figure that $\mathrm{LL}, \mathrm{RL}$ and the 5 lobes followed the same trend. The risk contribution fraction went up smoothly with the particle diameter and stabilized at a high level between $10 \mathrm{~nm}$ to $1 \mu \mathrm{m}$. Then a sharp decrease occurred after $1 \mu \mathrm{m}$ and the risk contribution fraction reduced to zero after $8 \mu \mathrm{m}$. These phenomena suggested that the fine particles $(10 \mathrm{~nm}-1 \mu \mathrm{m})$ could induce a higher potential health hazard. Right lung risk contribution fraction was about 1.8 times higher than that of left lung due to higher flow volume. The peak contribution fraction could reach $56 \%$ in the right lung. In the lung lobes, RLL faced the higher potential of carcinogenic risks as the risk contribution fraction was the highest among all lobes (Fig. 5(b)). The contribution fraction in RLL could reach as high as $28 \%$, accounting for half of the right lung. The RUL and LLL had nearly the same risk contribution fractions, up to $20 \%$ from $10 \mathrm{~nm}$ to $500 \mathrm{~nm}$. They all experienced a slight increase around 2-3 $\mu \mathrm{m}$ before the rapid decline. The maximum risk contribution in LUL was
$14.5 \%$, between the LLL and RML risk fractions. The risk contribution fraction in RML was at the lowest level among 5 lobes of less than $6 \%$. The risk contribution fraction trends in the trachea and main bronchi were drastically different from that in the lung lobes. The fraction peaked around $8 \%$ at $1 \mathrm{~nm}$ and then gradually fell to zero. Key features of the risk contribution fraction against the particle diameter for $\mathrm{Mn}\left(7440 \mathrm{~kg} \mathrm{~m}^{-3}\right)$ and $\mathrm{Ni}\left(8908 \mathrm{~kg} \mathrm{~m}^{-3}\right)$ were similar to that of $\mathrm{Cr}$ particles.

The mass diffusion coefficient $D$ is an important parameter describing nanoparticles transport, given as:

$$
D=\frac{k_{B} T C_{c}}{3 \pi \mu_{g} d_{p}}
$$

It is seen from Eq. (15) that smaller particles have a higher diffusion rate. Fig. 6 presented the risk contribution fractions of LL, RL, T\&M, LLL, LUL, RUL, RML, and RLL of nanoparticles from 1 to $500 \mathrm{~nm}$ against the mass diffusion coefficient and the fitted regression lines. The RCF in LL, RL, LLL, LUL, RUL, RML, and RLL remained stable when the mass diffusion coefficient is below $5 \times 10^{-7}$, and then a rapid drop occurred, while for the trachea and the main bronchi, the risk contribution fraction stayed at a low level when $D$ is less than $1.5 \times 10^{-7}$ and then rose. Based on the simulation, risk contribution fractions as a function of particle diffusivity $D\left(\mathrm{~m}^{2} \mathrm{~s}^{-1}\right)$ were developed. $\mathrm{D}^{-0.0096}, \mathrm{D}^{-0.0017}, \mathrm{D}^{0.3976}, \mathrm{D}^{-0.0091}, \mathrm{D}^{-0.0099}, \mathrm{D}^{-0.0011}, \mathrm{D}^{-0.0046}$, and $\mathrm{D}^{-0.0016}$ were found to provide the best fit for $\mathrm{LL}, \mathrm{RL}$, T\&M, LLL, LUL, RUL, RML, and RLL of nanoparticles from 1 to $500 \mathrm{~nm}$, at the breathing rate of $18 \mathrm{~L} \mathrm{~min}^{-1}$ (Fig. 6) The fitted empirical equations are:

$$
\begin{aligned}
& R C F_{L L-n m}=D^{-0.0096} \cdot \exp \left(3.05-5.4 \times 10^{5} D\right)+8.89 \\
& R C F_{R L-n m}=D^{-0.0017} \cdot \exp \left(3.65-4.51 \times 10^{5} D\right)+15.06 \\
& R C F_{T \& M-n m}=\left[1-0.992 \exp \left(-10.74 \cdot D^{0.3976}\right)\right] \times 100 \%
\end{aligned}
$$
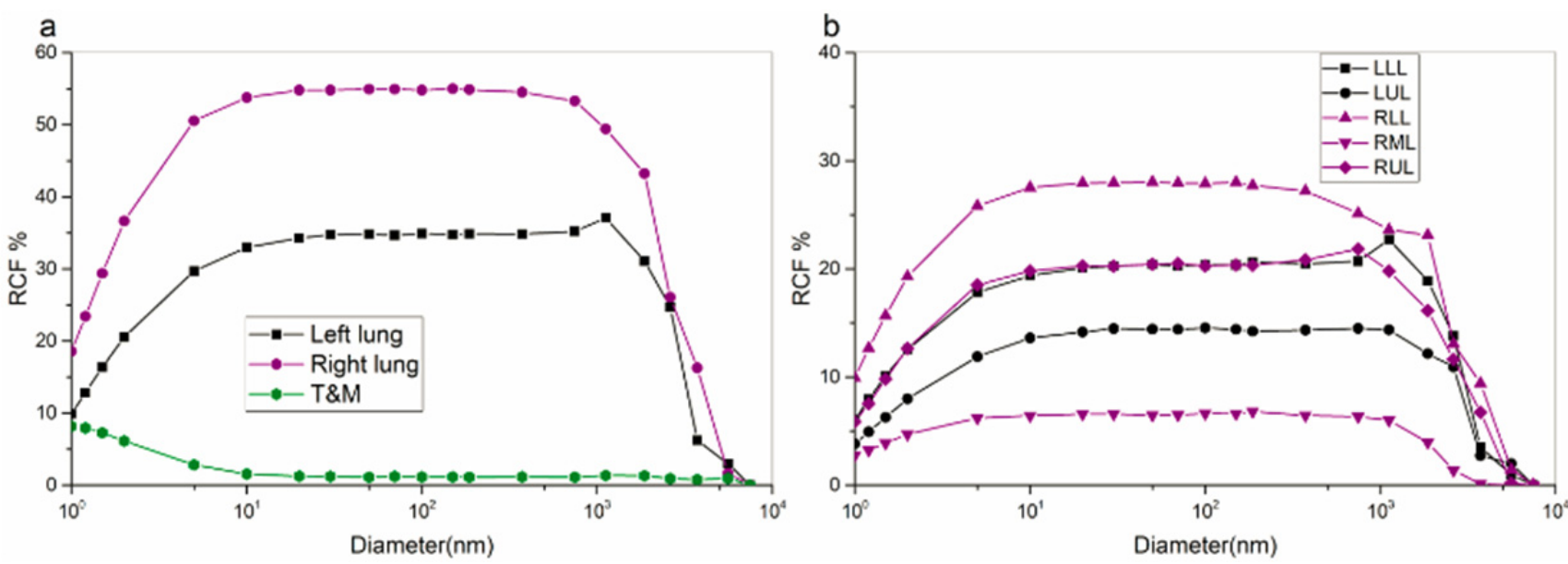

Fig. 5. Comparison of $\mathrm{Cr}$ particle $(1 \mathrm{~nm}-10 \mu \mathrm{m})$ risk contribution fraction against particle diameter: (a) left lung, right lung, trachea and main bronchi contribution fractions; (b) LLL, LUL, RLL, RML, RUL contribution fractions. 

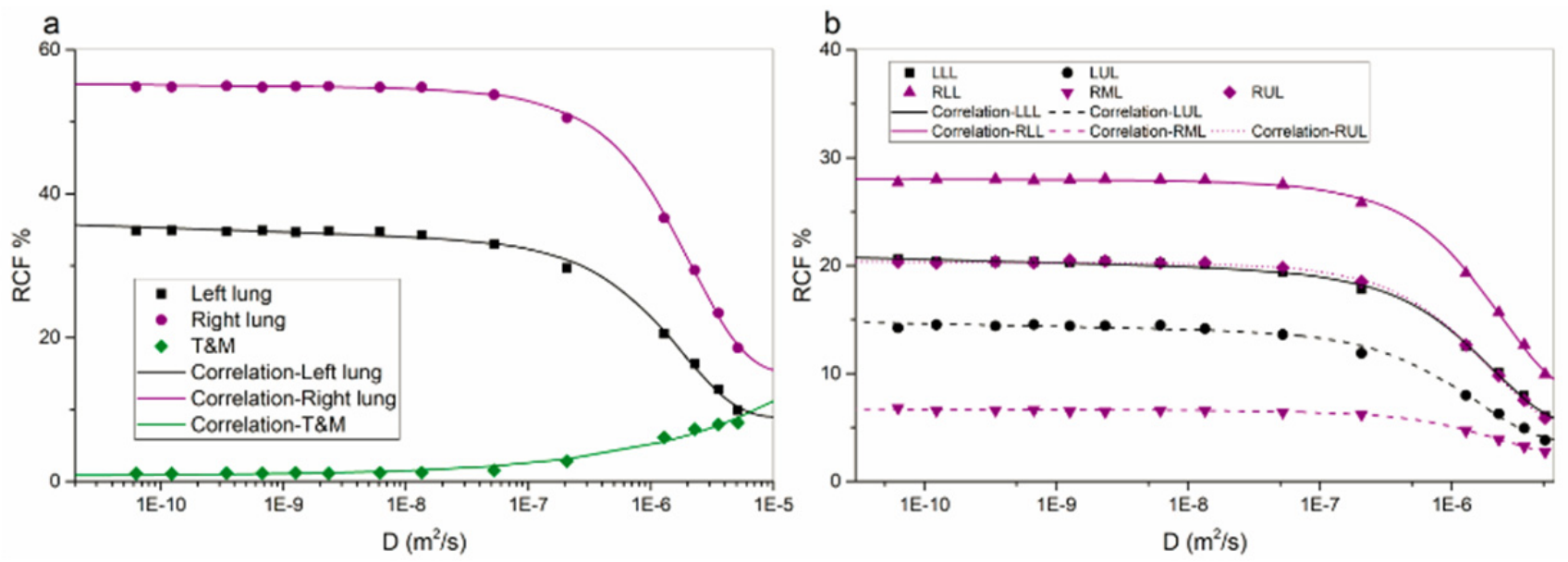

Fig. 6. Comparison of nanoparticles $(1 \mathrm{~nm}-500 \mathrm{~nm})$ risk contribution fraction: (a) left lung, right lung, trachea and main bronchi contribution fractions and fitted equations; (b) LLL, LUL, RLL, RML, RUL contribution fractions and fitted equations.

$$
\begin{aligned}
& R C F_{L L L-n m}=D^{-0.0091} \cdot \exp \left(2.53-4.77 \times 10^{5} D\right)+5.08 \\
& R C F_{L U L-n m}=D^{-0.0099} \cdot \exp \left(2.16-6.37 \times 10^{5} D\right)+3.71 \\
& R C F_{R L L-n m}=D^{-0.0011} \cdot \exp \left(2.98-4.14 \times 10^{5} D\right)+7.79 \\
& R C F_{R M L-n m}=D^{-0.0046} \cdot \exp \left(1.38-4.12 \times 10^{5} D\right)+2.25 \\
& R C F_{R U L-n m}=D^{-0.0016} \cdot \exp \left(2.7-5.09 \times 10^{5} D\right)+4.98
\end{aligned}
$$

The risk contribution fractions of LL, RL, T\&M, LLL, LUL, RUL, RML, and RLL for micron particles, ranging from $0.5 \mu \mathrm{m}$ to $10 \mu \mathrm{m}$ against the Stokes number together with the fitted equations, were plotted in Fig. 7. Except that the RCF in the trachea and main bronchi were stable at $1 \%$, all RCFs in LL, RL, LLL, LUL, RUL, RML, and RLL remained at a relatively high level and then fell at the Stokes number around 0.004 . The equations were developed as a function of the Stokes number $(S t)$, which correlated the deposition to length scale, particle density, size and flow rate related to inertial forces. The Stokes number is defined as:

$$
S t=\frac{\rho_{p} d_{p}{ }^{2} u}{18 \mu L}
$$

The fitted empirical equations, with the $r^{2}$ all above 0.95 , are as follows:

$$
\begin{aligned}
& R C F_{L L-\mu m}=35.38 \cdot \exp \left(-1477 S t^{2.11}\right) \\
& R C F_{R L-\mu m}=56.26 \cdot \exp \left(-40.97 S t^{1.07}\right) \\
& R C F_{T \& M-\mu m}=0.9176 S t^{-0.0304} \cdot \exp \left(-9.87 \times 10^{5} S t^{6.89}\right)
\end{aligned}
$$

$$
\begin{aligned}
& R C F_{L L L-\mu m}=21.14 \cdot \exp \left(-1422 S t^{2.08}\right) \\
& R C F_{L U L-\mu m}=14.22 \cdot \exp \left(-2058 S t^{2.24}\right) \\
& R C F_{R L L-\mu m}=27.23 \cdot \exp \left(-31.02 S t^{1.02}\right) \\
& R C F_{R M L-\mu m}=6.60 \cdot \exp \left(-90.51 S t^{1.63}\right) \\
& R C F_{R U L-\mu m}=21.19 \cdot \exp \left(-51.85 S t^{2.26}\right)
\end{aligned}
$$

Human has left and right lung. Each lung is composed of lobes, that left lung has 2 lobes and right lung has 3 lobes. It is of great significance to evaluate the risk contribution fraction in these lobes by studying particle deposition distribution. The risk contribution fraction in LL and RL were the sum of risk contribution fraction in LUL, LLL, and RLL, RML, RUL, respectively. Although the nanoand microparticle risk contribution fraction in lungs and lung lobes had the same form, the specific parameters were different in distinct regions. These differences were mainly due to the particle deposition distribution in current study. For nanoparticles, the index of the particle mass diffusivity in left lung, LLL and LUL were smaller than that in right lung, RUL, RML and RLL. While for microparticles, the index of the the Stokes number in left lung, LLL and LUL were larger than that in right lung, RUL, RML and RLL. The left lung and right lung index of the control parameter in particle risk contribution fraction were similar with that in LLL, LUL and RUL, RML, RLL, respectively.

\section{Health Risk Assessment}

The selected heavy metal particle risk contribution concentration in each lung lobe was an important parameter for evaluating health risk in the specific regions. The selected heavy metal particle risk contribution concentration in LLL was calculated by substituting Eqs. (19) and (28) 

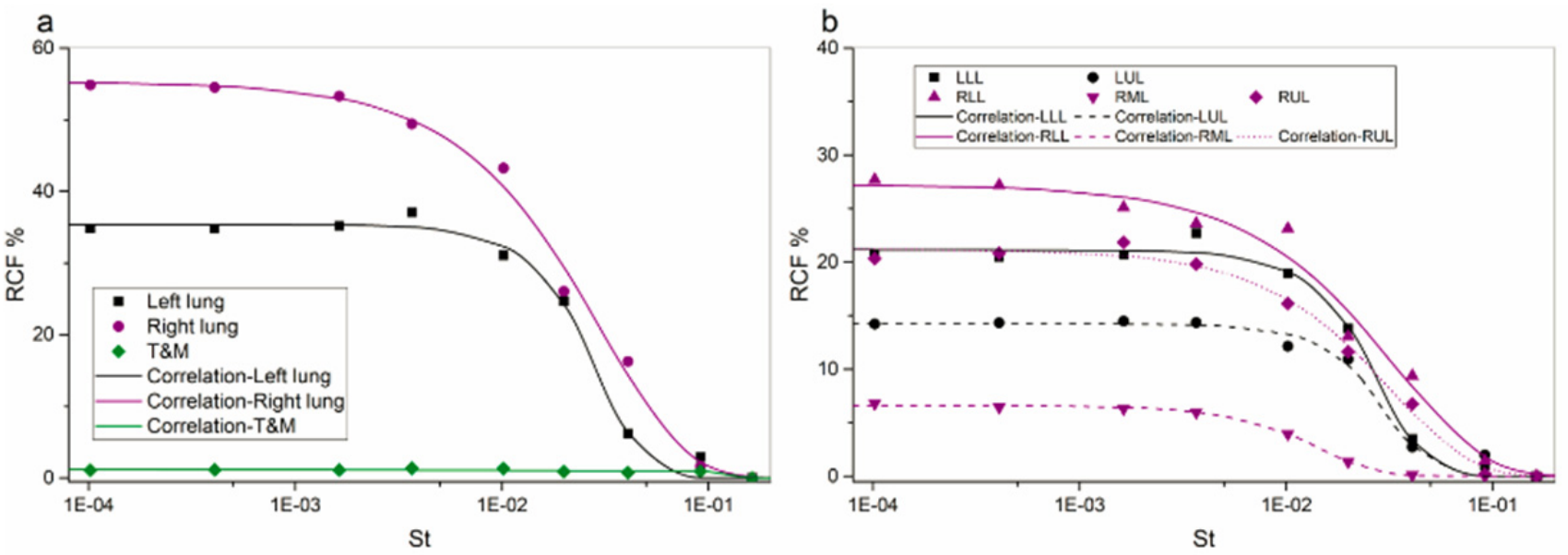

Fig. 7. Comparison of micron particle $(500 \mathrm{~nm}-10 \mu \mathrm{m})$ risk contribution fraction: (a) left lung, right lung, trachea and main bronchi contribution fractions and fitted equations; (b) LLL, LUL, RLL, RML, RUL contribution fractions and fitted equations.

into Eq. (7). Similarly, the risk contribution concentration in LUL, RUL, RML, RLL, LL, RL, and T\&M were obtained by substituting their RCFs into Eq. (7). The health risk assessment results for LLL, LUL, RUL, RML, RLL, LL, $\mathrm{RL}, \mathrm{T} \& \mathrm{M}$ and the whole respiratory system inhalation exposure to selected heavy metal elements $(\mathrm{Cr}, \mathrm{Mn}, \mathrm{Ni})$ in a bus stop scenario were given in Tables $2-4$. The results showed that most of the selected elements had the noncarcinogenic and carcinogenic risks within the limits (HQ $=1$ and $\mathrm{ELCR}=1 \times 10^{-6}$ ).

$\mathrm{Cr}, \mathrm{Mn}$, and $\mathrm{Ni}$ showed different non-carcinogenic and carcinogenic risks with varying size-dependent particle concentration, the inhalation risk, and the inhalation reference concentration. $\mathrm{Cr}$ poses a potential carcinogenic risk for people who commute by public transport, with the ELCR reaching $2.08 \times 10^{-5}$, exceeding the recommended limits. The ELCRs by Cr in LLL, LUL, RUL, RML, RLL, LL, and RL were also above the limit, suggesting high carcinogenic risks in the lungs. Ni posed the highest non-carcinogenic risk with the total inspiratory tract HQ at $5.73 \times 10^{-2}$, followed by $\mathrm{Cr}$ and $\mathrm{Mn}$, respectively. Although Ni had a relatively high non-carcinogenic risk, the potential carcinogenic risk was low. RLL had the highest health risk for the three selected elements, followed by LLL and RUL, which had similar level of potential risk. LUL and RML came the last.

Fig. 8 presented the potential risk contribution of LLL, LUL, RUL, RML, RLL, LL, RL, and T\&M with inhalation exposure to selected elements $(\mathrm{Cr}, \mathrm{Mn}, \mathrm{Ni})$ in a bus stop scene. For $\mathrm{Cr}, \mathrm{Mn}$, and $\mathrm{Ni}$, non-carcinogenic and carcinogenic health risk of the right lung were 1.5 times higher than the left lung. The trachea and main bronchi health risk contribution was less than $1 \%$ for all selected particles. The right lung risk contribution reached 50\% when exposed to $\mathrm{Cr}$, while the left lung contributed $33 \%$. The right and left lung risk contribution of $\mathrm{Ni}$ and $\mathrm{Mn}$ were $42.8 \%$ and $29.5 \%$, and $27.5 \%$ and $18.3 \%$, respectively. Inhalation risk contribution of $\mathrm{Cr}, \mathrm{Mn}, \mathrm{Ni}$ in the $\mathrm{TB}$ region were $84 \%, 46.7 \%$, and $73.4 \%$, respectively. Inhalation exposure to $\mathrm{Cr}$ posed a significantly higher risk than exposure to $\mathrm{Ni}$ and $\mathrm{Mn}$. On the other hand, the upper airway inhalation risk contribution of $\mathrm{Cr}, \mathrm{Mn}, \mathrm{Ni}$ were $16 \%, 53.3 \%$, and $26.6 \%$. It indicated that Mn could induce a higher risk in human upper airway than the other two elements. RLL posed $25.3 \%$, the highest health risk potential when facing $\mathrm{Cr}$ exposure, followed by LLL, RUL, LUL, and RML, of $19.3 \%, 18.9 \%, 13.5 \%$, and $15.9 \%$, respectively. Health risk potential for $\mathrm{Mn}$ and $\mathrm{Ni}$ exposure in the lung lobes were similar. The potential health risk in RLL was the highest, followed by LLL, RUL, LUL, and RML.

\section{CONCLUSION}

In this study, a regional health risk assessment was performed with a nasal-oral-tracheobronchial airway model. A bus stop exposure scenario using experimental data was applied to evaluate the non-carcinogenic and carcinogenic inhalation health risks for the trachea and main bronchi as well as the five lung lobes. The health consequences of $\mathrm{Cr}, \mathrm{Mn}$, and $\mathrm{Ni}$ exposure were measured in terms of the hazard quotient (HQ) and the excess lifetime cancer risk (ELCR). The results indicated significant particle deposition at the lung bifurcations, particularly on the inner sides, where the flow velocities were higher than those near the other sides. Fine particles $(10 \mathrm{~nm}-1 \mu \mathrm{m})$ consistently contributed the highest risk fraction for the LLL, LUL, RLL, RML, and RUL, suggesting potentially higher health consequences in the lungs from finer particles than coarser ones. For the right lung, the noncarcinogenic and carcinogenic risks were 1.5 times higher than for the left lung. Of the lung lobes, the RLL incurred the highest risk, while the RML incurred the lowest. In the experimental scenario, $\mathrm{Cr}$ posed a carcinogenic lung risk to people who commuted by public transport, with the ELCRs of the lungs and the five lung lobes all exceeding the recommended limits. The inhalation risk contributions of $\mathrm{Cr}, \mathrm{Mn}$, and $\mathrm{Ni}$ to the $\mathrm{TB}$ region were $84 \%, 46.7 \%$, and $73.4 \%$, respectively; however, the health 
Table 2. Health risks for inhalation exposure to Cr at LLL, LUL, RLL, RML, RUL, LL, RL, and T\&M in a bus stop scene.

\begin{tabular}{llllllll}
\hline Risk assessment & LLL & LUL & RLL & RML & RUL & T\&M & Inhalation \\
\hline HQ $^{\mathrm{a}}$ & $1.11 \times 10^{-3}$ & $7.80 \times 10^{-4}$ & $1.46 \times 10^{-3}$ & $3.42 \times 10^{-4}$ & $1.09 \times 10^{-3}$ & $5.68 \times 10^{-5}$ & $5.78 \times 10^{-3}$ \\
& $1.90 \times 10^{-3}$ & & $2.90 \times 10^{-3}$ & & & & \\
ELCR $^{\mathrm{a}}$ & $4.01 \times 10^{-6}$ & $2.81 \times 10^{-6}$ & $5.27 \times 10^{-6}$ & $1.23 \times 10^{-6}$ & $3.93 \times 10^{-6}$ & $2.04 \times 10^{-7}$ & $2.08 \times 10^{-5}$ \\
& $6.82 \times 10^{-6}$ & & $1.04 \times 10^{-5}$ & & & &
\end{tabular}

Table 3. Health risks for inhalation exposure to Mn at LLL, LUL, RLL, RML, RUL, LL, RL, and T\&M in a bus stop scene.

\begin{tabular}{llllllll}
\hline Risk assessment & LLL & LUL & RLL & RML & RUL & T\&M & Inhalation \\
\hline $\mathrm{HQ}^{\mathrm{a}}$ & $1.27 \times 10^{-4}$ & $8.92 \times 10^{-5}$ & $1.67 \times 10^{-4}$ & $3.25 \times 10^{-5}$ & $1.27 \times 10^{-4}$ & $9.99 \times 10^{-6}$ & $1.18 \times 10^{-3}$ \\
& $2.16 \times 10^{-4}$ & & $3.24 \times 10^{-4}$ & & & \\
\hline
\end{tabular}

Table 4. Health risks for inhalation exposure to Ni at LLL, LUL, RLL, RML, RUL, LL, RL, and T\&M in a bus stop scene.

\begin{tabular}{llllllll}
\hline Risk assessment & LLL & LUL & RLL & RML & RUL & T\&M & Inhalation \\
\hline $\mathrm{HQ}^{\mathrm{a}}$ & $1.00 \times 10^{-2}$ & $6.92 \times 10^{-3}$ & $1.23 \times 10^{-2}$ & $2.73 \times 10^{-3}$ & $9.56 \times 10^{-3}$ & $5.73 \times 10^{-4}$ & $5.73 \times 10^{-2}$ \\
& $1.69 \times 10^{-2}$ & & $2.46 \times 10^{-2}$ & & & & \\
ELCR $^{\mathrm{a}}$ & $1.56 \times 10^{-8}$ & $1.08 \times 10^{-8}$ & $1.92 \times 10^{-8}$ & $4.25 \times 10^{-9}$ & $1.49 \times 10^{-8}$ & $8.94 \times 10^{-10}$ & $8.94 \times 10^{-8}$ \\
& $2.64 \times 10^{-8}$ & & $3.83 \times 10^{-8}$ & & & & \\
\hline
\end{tabular}

${ }^{\mathrm{a}}$ The acceptable risk limits for HQ and ELCR are 1 and $10^{-6}$, respectively.

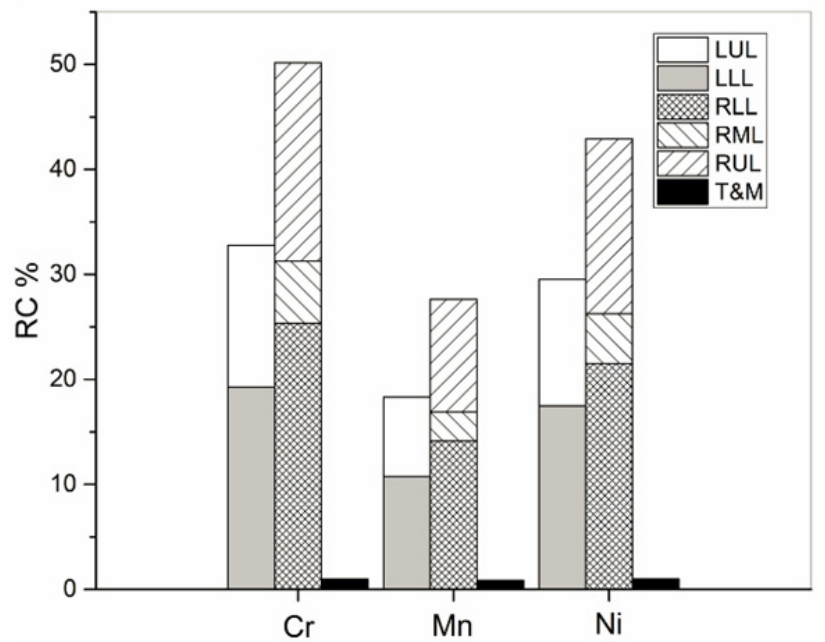

Fig. 8. Risk contribution of LLL, LUL, RLL, RML, RUL, $\mathrm{LL}, \mathrm{RL}$, and T\&M for inhalation exposure to the selected elements $(\mathrm{Cr}, \mathrm{Mn}, \mathrm{Ni})$ in a bus stop scene.

risk to the trachea and main bronchi contributed less than $1 \%$ regardless of the element. The risk of developing lung disease from inhalation exposure was significantly higher with $\mathrm{Cr}$ than with $\mathrm{Ni}$ and $\mathrm{Mn}$. However, of the three elements, Mn potentially induced the highest chance of developing upper respiratory disease.

This study was also subject to several limitations. Firstly, the airway model was based on CT scans of a single human subject. Because real airways vary based on the gender, age, and physical health of the individual, our estimation of the regional health risk, which depends on the particle deposition distribution, may not be universally applicable. Secondly, local particle deposition may be influenced by the breathing pattern. Finally, airway humidity, which also affects particle deposition, was not addressed in this work. Future health risk assessments should be performed with a standardized airway model and consider the factors of transient breathing cycles and airway humidity.

\section{ACKNOWLEDGMENTS}

This study was supported by National Natural Science Foundation of China (Grant No. 51706123 and 91643102), Ministry of Science and Technology of the People's Republic of China (Grant No. 2016YFC0802801 and 2016YFC0802807), National Science Fund for Distinguished Young Scholars of China (Grant No. 71725006), Australian Research Council (Project ID: DP160101953 and DE180101138) and China Scholarship Council (Student No. 201706210128). The authors are deeply grateful to these supports.

\section{REFERENCES}

Balásházy, I., Hofmann, W. and Heistracher, T. (2003). Local particle deposition patterns may play a key role in the development of lung cancer. J. Appl. Physiol. 94: 1719-1725.

Baxter, C.S., Ross, C.S., Fabian, T., Borgerson, J.L., Shawon, J., Gandhi, P.D., Dalton, J.M. and Lockey, J.E. (2010). Ultrafine particle exposure during fire suppression-Is it an important contributory factor for coronary heart disease in firefighters? J. Occup. Environ. Med. 52: 791-796.

Bell, M.L., Dominici, F., Ebisu, K., Zeger, S.L. and Samet, J.M. (2007). Spatial and temporal variation in $\mathbf{P M}_{2.5}$ chemical composition in the United States for health effects studies. Environ. Health Perspect. 115: 989-995.

Deng, Q., Ou, C., Chen, J. and Xiang, Y. (2018). Particle 
deposition in tracheobronchial airways of an infant, child and adult. Sci. Total Environ. 612: 339-346.

Donaldson, K., Stone, V., Seaton, A. and MacNee, W. (2001). Ambient particle inhalation and the cardiovascular system: Potential mechanisms. Environ. Health Perspect. 1094: 523-527.

U.S. EPA (2009). Risk assessment guidance for superfund Volume I: Human Health Evaluation Manual (Part F, supplemental guidance for inhalation risk assessment). https://www.epa.gov/risk/risk-assessment-guidance-sup rfund-rags-part-f, Last Access: 30 June 2018.

U.S. EPA (2018). Regional Screening Levels (RSLs) Generic tables. https://www.epa.gov/risk/regional-scree ning-levels-rsls-generic-tables, Last Access: 30 June 2018.

Ferreira-Baptista, L. and De Miguel, E. (2005). Geochemistry and risk assessment of street dust in Luanda, Angola: A tropical urban environment. Atmos. Environ. 39: 45014512.

Franklin, M., Koutrakis, P. and Schwartz, J. (2008). The role of particle composition on the association between $\mathrm{PM}_{2.5}$ and mortality. Epidemiology 19: 680-689.

Häußermann, S., Bailey, A.G., Bailey, M.R., Etherington, G. and Youngman, M. (2002). The influence of breathing patterns on particle deposition in a nasal replicate cast. J. Aerosol Sci. 33: 923-933.

Horsfield, K., Dart, G., Olson, D.E., Filley, G.F. and Cumming, G. (1971). Models of the human bronchial tree. J. Appl. Physiol. 31: 207-217.

Inthavong, K., Choi, L., Tu, J., Ding, S. and Thien, F. (2010). Micron particle deposition in a tracheobronchial airway model under different breathing conditions. Med. Eng. Phys. 32: 1198-1212.

Inthavong, K., Ge, Q.J., Li, X.D. and Tu, J.Y. (2012). Detailed predictions of particle aspiration affected by respiratory inhalation and airflow. Atmos. Environ. 62: 107-117.

Kim, M., Collier, G.J., Wild, J.M. and Chung, Y.M. (2018). Effect of upper airway on tracheobronchial fluid dynamics. Int. J. Numer. Methods Biomed. Eng. 34: e3112.

Kong, S., Lu, B., Bai, Z., Zhao, X., Chen, L., Han, B., Li, Z., Ji, Y., Xu, Y., Liu, Y. and Jiang, H. (2011). Potential threat of heavy metals in re-suspended dusts on building surfaces in oilfield city. Atmos. Environ. 45: 4192-4204.

Lizal, F., Belka, M., Adam, J., Jedelsky, J. and Jicha, M. (2015). A method for in vitro regional aerosol deposition measurement in a model of the human tracheobronchial tree by the positron emission tomography. Proc. Inst. Mech. Eng. H 229: 750-757.

Lovett, C., Shirmohammadi, F., Sowlat, M.H. and Sioutas, C. (2018). Commuting in Los Angeles: Cancer and noncancer health risks of roadway, light-rail and subway transit routes. Aerosol Air Qual. Res. 18: 2363-2374.

Loxham, M., Cooper, M.J., Gerlofs-Nijland, M.E., Cassee, F.R., Davies, D.E., Palmer, M.R. and Teagle, D.A.H. (2013). PPhysicochemical characterization of airborne particulate matter at a mainline underground railway station. Environ. Sci. Technol. 47: 3614-3622.

Menter, F.R., Langtry, R. and Völker, S. (2006). Transition modelling for general purpose CFD codes. Flow Turbul. Combust. 77: 277-303.

Sah, D., Verma, P.K., Kumari, K.M. and Lakhani, A. (2017). Chemical partitioning of fine particle-bound As, $\mathrm{Cd}, \mathrm{Cr}, \mathrm{Ni}, \mathrm{Co}, \mathrm{Pb}$ and assessment of associated cancer risk due to inhalation, ingestion and dermal exposure. Inhalation Toxicol. 29: 483-493.

Schlesinger, R.B., Bohning, D.E., Chan, T.L. and Lippmann, M. (1977). Particle deposition in a hollow cast of the human tracheobronchial tree. J. Aerosol Sci. 8: 429-445.

Schünke, M., Schulte, E., Ross, L.M., Lamperti, E.D. and Schumacher, U. (2006). Atlas of anatomy: Neck and internal organs, Thieme.

Se, C., Inthavong, K. and Tu, J. (2010). Unsteady particle deposition in a human nasal cavity during inhalation. $J$. Comput. Multiphase Flows 2: 207-218.

Tian, L. and Ahmadi, G. (2012). Transport and deposition of particles in the upper tracheobronchial tree were analyzed using a multi-level asymmetric lung bifurcation model. J. Comput. Multiphase Flows 4: 159-182.

Tian, L. and Ahmadi, G. (2016). Transport and deposition of nano-fibers in human upper tracheobronchial airways. J. Aerosol Sci. 91: 22-32.

Tian, L., Shang, Y., Chen, R., Bai, R., Chen, C., Inthavong, K. and Tu, J. (2017). A combined experimental and numerical study on upper airway dosimetry of inhaled nanoparticles from an electrical discharge machine shop. Part. Fibre Toxicol. 14: 24.

Turner, M.C., Krewski, D., Pope, C.A., Chen, Y., Gapstur, S.M. and Thun, M.J. (2011). Long-term ambient fine particulate matter air pollution and lung cancer in a large cohort of never-smokers. Am. J. Respir. Crit. Care 184: 1374-1381.

Vu, T.V., Beddows, D.C., Delgado-Saborit, J.M. and Harrison, R.M. (2016). Source apportionment of the lung dose of ambient submicrometre particulate matter. Aerosol Air Qual. Res. 16: 1548-1557.

Weibel, E.R. (1963). Morphometry of the human lung. Springer, Berlin, Heidelberg.

Xu, X., Shang, Y., Tian, L., Weng, W. and Tu, J. (2018). A numerical study on firefighter nasal airway dosimetry of smoke particles from a realistic composite deck fire. J. Aerosol Sci. 123: 91-104.

You, S., Yao, Z., Dai, Y. and Wang, C. (2017). A comparison of PM exposure related to emission hotspots in a hot and humid urban environment: Concentrations, compositions, respiratory deposition, and potential health risks. Sci. Total Environ. 599-600: 464-473.

Zhai, Y., Liu, X., Chen, H., Xu, B., Zhu, L., Li, C. and Zeng, G. (2014). Source identification and potential ecological risk assessment of heavy metals in $\mathrm{PM}_{2.5}$ from Changsha. Sci. Total Environ. 493: 109-115.

Zhang, Z., Kleinstreuer, C. and Kim, C.S. (2008). Airflow and nanoparticle deposition in a 16-generation tracheobronchial airway model. Ann. Biomed. Eng. 36: 2095-2110.

Zhang, Z. and Kleinstreuer, C. (2011). Laminar-to-turbulent fluid-nanoparticle dynamics simulations: Model 
comparisons and nanoparticle-deposition applications. Int. J. Numer. Methods Biomed. Eng. 27: 1930-1950.

Zheng, J., Zhan, C., Yao, R., Zhang, J., Liu, H., Liu, T., Xiao, W., Liu, X. and Cao, J. (2018). Levels, sources, markers and health risks of heavy metals in $\mathrm{PM}_{2.5}$ over a typical mining and metallurgical city of central China. Aerosol Sci. Eng. 2: 1-10.

Zheng, N., Liu, J., Wang, Q. and Liang, Z. (2010). Heavy metals exposure of children from stairway and sidewalk dust in the smelting district, northeast of China. Atmos. Environ. 44: 3239-3245.

Zheng, X., Xu, X., Yekeen, T.A., Zhang, Y., Chen, A., Kim, S.S., Dietrich, K.N., Ho, S., Lee, S., Reponen, T. and Huo, X. (2016). Ambient air heavy metals in $\mathrm{PM}_{2.5}$ and potential human health risk assessment in an informal electronic-waste recycling site of China. Aerosol Air Qual. Res. 16: 388-397.

Zhou, Y. and Cheng, Y.S. (2005). Particle deposition in a cast of human tracheobronchial airways. Aerosol Sci. Technol. 39: 492-500.

Received for review, November 6, 2018

Revised, February 25, 2019

Accepted, April 1, 2019 